\title{
Die oberen Atemwege im Speziesvergleich
}

13. Workshop des Arbeitskreises „Vergleichende Pathologie und Pathophysiologie des Respiratorischen Systems“ (VPPRS) der Deutschen Gesellschaft für Veterinärmedizin (DVG) in Kooperation mit der Sektion 10 der Deutschen Gesellschaft für Pneumologie und Beatmungsmedizin (DGP) (im Rahmen des 51. Kongresses der DGP)

\author{
Species-Specific Comparison of Upper Airways
}

Autoren

P. Reinhold ${ }^{1}$, S. Grützenmacher ${ }^{2}$, R. Pabst ${ }^{3}$, R. Koch ${ }^{4}$, R. Schulz ${ }^{5}$, N. Kirschvink ${ }^{6}$, G. U. Oechtering ${ }^{7}$, J. P. Lippert ${ }^{7}$, K. Fey ${ }^{8}$, M. Rosenbruch ${ }^{9}$

Institute

1 Institut für molekulare Pathogenese im Friedrich-LoefflerInstitut (Bundesinstitut für Tiergesundheit)

2 Dietrich-Bonhoeffer Klinikum Neubrandenburg

3 Medizinische Hochschule

Hannover

${ }^{4}$ Tierärztliche Hochschule Hannover

5 Universitätsklinik Marburg und Gießen

${ }^{6}$ University of Namur (Belgium) ${ }^{7}$ Veterinärmedizinische Fakultät der Universität Leipzig

${ }^{8}$ Justus-Liebig-Universität Gießen

${ }_{9}^{9}$ Bayer Schering Pharma AC
Bibliografie

DOI http://dx.doi.org/ 10.1055/s-0030-1255517

Pneumologie 2010; 64: 442-455 @ Georg Thieme Verlag KG Stuttgart · New York ISSN 0934-8387

\section{Korrespondenzadresse}

\section{PD Dr. Dr. Petra Reinhold}

Friedrich-Loeffler-Institut

(Bundesforschungsinstitut für

Tiergesundheit)

Naumburger Str. 96 a

07743 Jena

petra.reinhold@fli.bund.de

\section{Editorial}

Werden im Rahmen wissenschaftlicher Kongresse Themen des Respirationstraktes behandelt, so steht bei der überwiegenden Mehrzahl von Veranstaltungen die Lunge im Vordergrund. Allerdings dürfen die oberen Atemwege keinesfalls als „nebensächlich“ betrachtet oder hintan gestellt werden. Im Respirationstrakt sind sie morphologisch und funktionell der Lunge voran gestellt und für wesentliche Körperfunktionen, z.B. das Riechen und die Stimmbildung, verantwortlich. Eine ungestörte Funktion dieser Organe, von der Nase über den Larynx bis zur Trachea - z. B. als primärer Filter der Atemluft - trägt auch zu einer kontinuierlichen Sicherstellung des Gasaustauschs bei.

Der Arbeitskreis „Vergleichende Pathologie und Pathophysiologie des Respiratorischen Systems“ hat bei seinem 13. Workshop am 17. März 2010 in Hannover diesen Gegebenheiten Rechnung getragen und „Die oberen Atemwege im Speziesvergleich" als übergeordnetes Thema behandelt.

Den Teilnehmern wurde dabei ein weites Spektrum an Zusammenhängen und Details über verschiedene Tierarten und den Menschen dargestellt. In den Vorträgen wurden sowohl experimentell relevante Spezies wie Ratte und Maus berücksichtigt, wie auch landwirtschaftliche Nutztiere (Schwein und Rind) und Liebhabertierarten (Hund und Pferd).
Vergleichende Darstellungen behandelten die Anatomie und Physiologie von Nase, Kehlkopf und Stimmapparat in Gegenüberstellungen Mensch - Paarhufer (Wildwiederkäuer, Schweine) sowie das Mukosa-assoziierte lymphatische System der Nase und des Larynx im Vergleich Nager - Mensch.

Verschiedenste Betrachtungsweisen zu den Strömungswiderständen der oberen Atemwege belegten nicht nur spezies-spezifische Besonderheiten, sondern verdeutlichten auch Ähnlichkeiten in der Komplexität obstruktiver Funktionsstörungen an brachycepahlen Hunden oder Katzen, Menschen mit obstruktiver Schlafapnoe oder Pferden unter dem Einfluss von Sedativa. Moderne diagnostische Methoden der Bildgebung und Funktionsdiagnostik veranschaulichten den $\mathrm{Zu}$ sammenhang zwischen strukturellen und funktionellen Veränderungen und verdeutlichten die nutzbringenden Effekte von laserassistierten operativen Öffnungen der oberen Atemwege in Form einer signifikanten Reduktion der nasalen Strömungswiderstände.

Insgesamt hat auch dieser Workshop des Arbeitskreises „Vergleichende Pathologie und Pathophysiologie des Respiratorischen Systems“ wieder gezeigt, dass sich Human- und Veterinärmedizin bei der Bearbeitung zahlreicher Fragestellungen hervorragend ergänzen und speziesübergreifende Diskussionen die Arbeit der jeweils anderen Disziplin durch Anregungen und Ideen bereichern können.

M. Rosenbruch, P. Reinhold 


\section{Betrachtungen zur Anatomie und Physiologie von Säugetiernasen}

\section{S. Grützenmacher}

Klinik für Hals-Nasen- und Ohrenheilkunde, Kopf- und

Halschirurgie, Plastische Operationen

Dietrich-Bonhoeffer Klinikum, Neubrandenburg

\section{Zusammenfassung}

$\nabla$

Die Nasen der Säugetiere weisen eine anatomisch und strömungsphysiologisch relativ klare Trennung zwischen Regio respiratoria und Regio olfactoria auf. Beim Menschen rotieren die olfactorischen Muscheln (Ethmoturbinalia) durch die erfolgte Schädelbasisknickung (klinorhynchi) in die respiratorische Region und übernehmen auch respiratorische Aufgaben.

Voraussetzung für die respiratorische Funktion der Nase ist ein intensiver Kontakt zwischen Atemluft und Schleimhaut. Dieser Kontakt findet bei allen untersuchten Säugetierspezies im Funktionsbereich (Muschelregion/Regio respiratoria) statt.

Beim Menschen bilden die Nasenabschnitte vom Ostium nasale externum bis zum Kopf der unteren Muschel den Einströmbereich. Er verteilt die Strömung über den gesamten Nasenquerschnitt und erzeugt Turbulenzen. Der Einströmbereich des Menschen kann als Anpassung an die relative Höhenzunahme der Nase betrachtet werden (Längen-Höhen-Relation).

\section{Abstract \\ $\nabla$}

From the anatomical and flow dynamic point of view, the mammalian noses are strictly divided in a respiratory and olfactory area. In humans, the middle and the superior turbinate (ethmoturbinates) fulfill the respiratory and olfactory function.

An intensive contact between breathing air and respiratory mucosa is the most important pre-condition for the respiratory function of the nose. In all investigated species this contact takes place in the functional area of the nose, i.e. the area of the turbinates.

In humans, the area from the external nasal ostium up to the head of the inferior turbinate is called „inflow area“. This part of the nose distributes the airflow over the complete nasal cross sectional area and generates turbulent flow. The inflow area in the human nose is an adaptation to the relatively increased nasal height during evolution.

\section{Vorbetrachtungen \\ $\nabla$}

Die Hauptaufgabe der Nase besteht im Reinigen, Anfeuchten und Temperieren der Atemluft [1,2]. Die phylogenetisch älteste Funktion der Nase ist das Riechen [3]. Es ist deshalb anzunehmen, dass im Laufe der Evolution eine Anpassung der Nase an die „neue“ Aufgabe, die Respiration, erfolgte.

Für die Klimatisierungsfunktion muss die Nase den Stoff- und Energieaustausch mit der Atemluft realisieren, was letztlich durch ein labiles Gleichgewicht zwischen Luftströmung und Nasenschleimhaut möglich ist. Eine ausreichend turbulente und über den gesamten Nasenquerschnitt verteilte Luftströmung ist die Voraussetzung für den Kontakt zwischen den Luftteilchen und der respiratorischen Schleimhaut. Ein niedriger nasaler Atemwiderstand ermöglicht eine kontinuierliche Nasenatmung. Eine gesunde und schwellfähige respiratorische Nasenschleimhaut ist die Voraussetzung für den Stoff- und Energieaustausch mit den strömenden Luftpartikeln.

Die notwendige Schleimhautregeneration wird dabei durch die Energierückgewinnung in der Exspirationsphase, durch den Nasenzyklus und durch den mukoziliaren Transport unterstützt $[4,5]$.

An diese komplexen Vorgänge ist die Nase morphologisch angepasst. Es ist bekannt, dass bereits kleine Formabweichungen oder anatomische Variationen eine gestörte respiratorische Funktion verursachen können.

\section{Anatomische Betrachtungen \\ $\nabla$}

Bei der überwiegenden Anzahl der Säugetierspezies findet man jeweils zwei Conchae im direkten Atemstrom (Maxilloturbinale oder Concha nasalis ventralis und Nasoturbinale oder Concha nasalis dorsalis), und zusätzlich eine unterschiedliche Anzahl von Conchae (Ethmoturbinalia) oberhalb des Ductus nasopharyngeus. Die Ethmoturbinalia und das Nasoturbinale sind überwiegend mit olfaktorischem Epithel (Regio olfactoria) besetzt und werden vom Os ethmoidale gebildet. Sie bilden die Regio olfactoria, welche nach kaudal knöchern vom Ductus nasopharyngeus getrennt ist [6]. In Analogie zum Menschen entsprechen diese Muscheln der Concha nasalis media und superior [7]. Gelegentlich findet sich beim Menschen eine Concha nasalis suprema (Concha santorini). Eine Abgrenzung zum Ductus nasopharyngeus existiert hier nicht.

Die größte respiratorische Concha der Säugetiere, die Concha nasalis ventralis (Maxilloturbinale), beginnt direkt an der lateralen Wand des Ostium nasale externum. Sie besitzt in Analogie zur unteren Muschel des Menschen ein eigenes Os turbinale [6].

Die Schädelbasis ist der verbindende Teil zwischen Schädeldach und Gesichtsschädel und bildet einen wesentlichen Anteil der oberen und hinteren Grenzen der Nasenhöhle.

Beim Menschen kommt es aufgrund der Entwicklung des aufrechten Gangs und der erheblichen Zunahme des Hirnvolumens zu einer Beugung der Schädelbasis (klinorhynchi) [8]. Hierdurch rotiert die bei den Tierspezies eher vertikal verlaufende Lamina cribrosa in eine horizontale Position. Weiterhin kommt es durch die Änderung der Größenverhältnisse zwischen Hirn- und Gesichtsschädel beim Menschen zu einer relativen Verkürzung des Mittelgesichts, was sich in der Längen-Höhen-Relation der Nase ausdrücken lässt.

\section{Strömungsphysiologische Betrachtungen}

Während die nasale Atemströmung des Menschen umfassend untersucht worden ist, finden sich zur nasalen Atemphysiologie weiterer Säugetierspezies nur wenige Hinweise in der Literatur. Eigene strömungsphysikalische Untersuchungen an Nasenmodellen von Menschen und verschiedenen Paarhuferspezies zeigen folgenden Verlauf der Atemströmung in der Nase: Beim Menschen wird das gesamte Cavum nasi durchströmt. Im Bereich der Nasenmuscheln ist die Strömung turbulent, diese Region wird als „Funktionsbereich“ bezeichnet. Der Bereich vom Ostium nasale externum bis zum Kopf der unteren Muschel bildet den „Einströmbereich“. Er verteilt die Strömung über den gesamten Nasenquerschnitt und generiert Turbulenzen. Der Einströmbereich 

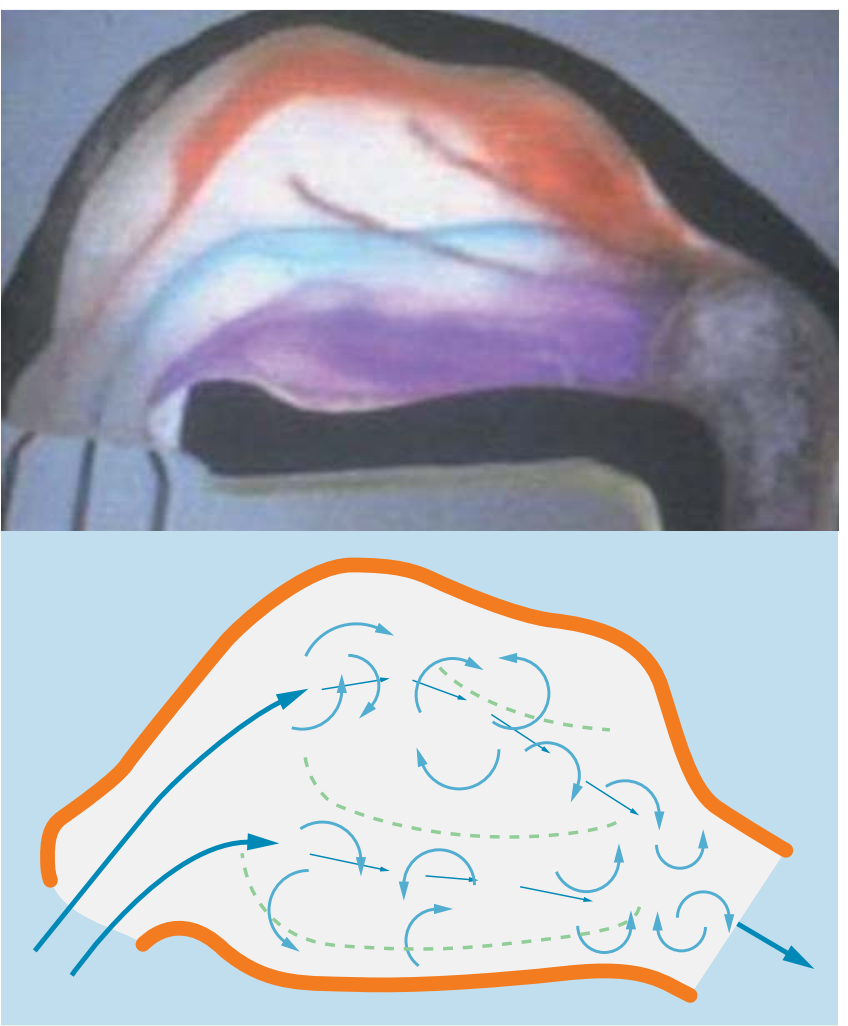

Abb. 1 Strömungsverlauf in der Nase eines Menschen bei einem Flow von $200 \mathrm{ml} / \mathrm{s}$.
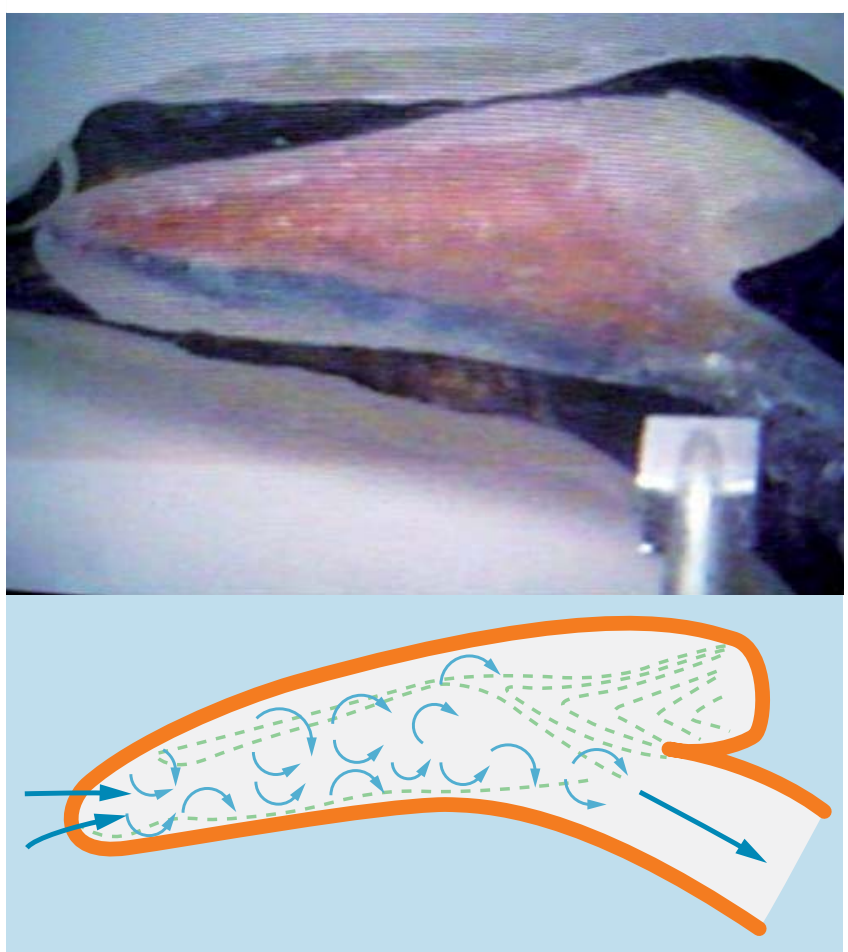

Abb. 2 Strömungsverlauf in der Nase eines Paarhufers (Schaf) bei einem Flow von $200 \mathrm{ml} / \mathrm{s}$. ist im Wesentlichen in der äußeren Nase untergebracht $[9,10]$ (๑ Abb. 1).

Bei Paarhufernasen verläuft die Atemströmung über die Concha nasalis dorsalis und ventralis. Die Luftströmung ist im gesamten Verlauf turbulent. Ein Einströmbereich, wie beim Menschen, existiert nicht. Die Regio olfactoria mit den Ethmoturbinalia ist nur gering durchströmt. Sie bildet einen sogenannten Totraum mit Kriechströmungen und Wirbelbildung [11] ( $\bullet$ Abb. 2).

Bei diesen Tierspezies setzt die ventrale Muschel unmittelbar am Ostium nasale externum an, so dass die gesamte Nasenhöhle ab dem Ostium nasale externum als Funktionsbereich (Region der Nasenmuscheln) anzusehen ist.

Da bei den untersuchten Tierspezies ein „Einströmbereich“ fehlt, ist $\mathrm{zu}$ vermuten, dass er und somit auch die äußere Nase des Menschen eine Anpassung an das für den Menschen typische kurze und hohe Cavum nasi darstellt.

\section{Literatur}

1 Drettner B, Falck B, Simon H. Measurements of the air conditioning capacity of the nose during normal and pathological conditions and pharmacological influence. Acta Otolaryngol 1977; 84: 266 - 277

2 Keck T, Leiacker R, Lindemann J, Rettinger G, Kuhnemann S. Endonasales Temperatur- und Feuchteprofil nach Exposition zu verschieden klimatisierter Einatemluft. HNO 2001; 49: 372 - 377

3 Hooton EA. Up from the ape. New York: Mac Millan; 1931

4 Toremalm NG. Aerodynamics and mucociliary function of upper airways. Eur J Respir Dis Suppl 1985; 139: 54-56

5 Soane RJ, Carney AS, Jones NS et al. The effect of the nasal cycle on mucociliary clearance. Clin Otolaryngol 2001; 26: 9-15

6 Seydel 0 . Über die Nasenhöhle der höheren Säugethiere und des Menschen. In: Morphologisches Jahrbuch. Bd. 17. Leipzig: Wilhelm Engelmann Verlag; 1891: 44-99

7 Zuckerkandl E. Normale und Pathologische Anatomie der Nasenhöhle und ihrer pneumatischen Anhänge. Wien: Braumüller; 1882-92

8 Schuhmacher GH. Anatomie für Zahnmediziner. Heidelberg: Hüthig; 1997

9 Mlynski G, Grützenmacher S, Plontke S et al. Correlation of nasal morphology and respiratory function. Rhinology 2001; 39: 197-201

10 Grützenmacher S, Robinson DM, Lang C et al. Investigations of the influence of external nose deformities on nasal airflow. ORL J Otorhinolaryngol Relat Spec 2005; 67: 154-159

11 Grützenmacher S, Robinson DM, Sevecke J et al. Comparative investigations of anatomy and physiology in mammalian noses (Homo sapiens - Artiodactyla). Rhinology 2010; 48: accepted for publication

\section{Bibliografie}

DOI $10.1055 / \mathrm{s}-0029-1244254$

Pneumologie 2010; 64; 443-455

(c) Georg Thieme Verlag KG Stuttgart · New York

ISSN 0934-8387

\section{Korrespondenzadresse}

\section{PD Dr. med. habil. Stefan Grützenmacher}

Klinik für Hals-Nasen- und Ohrenheilkunde, Kopf- und Halschirurgie, Plastische Operationen Dietrich-Bonhoeffer-Klinikum

Salvador-Allendestraße 30

17036 Neubrandenburg

hno@dbknb.de 


\section{Das lymphatische Gewebe der Nase (NALT) und des Kehlkopfes (LALT) im Speziesvergleich: Mensch, Ratte, Maus}

\section{R. Pabst}

Institut für Immunmorphologie, Medizinische Hochschule Hannover

\section{Zusammenfassung}

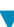

Bezüglich des Nasen- und Larynx-assoziierten lymphatischen Gewebes (NALT und LALT) bestehen deutliche Unterschiede zwischen Mensch, Ratte und Maus. Während bei Ratte und Maus NALT eine paarige Ansammlung von lymphatischem Gewebe in der Nase darstellt, sind beim Menschen einzelne schleimhautassoziierte Lymphfollikel in allen Bereichen der Nase zu finden. Zusätzlich besitzt der Mensch Tonsillen, die bei Ratte und Maus nicht existieren. Im Bereich des Kehlkopfes ist beim Menschen, nicht aber bei der Ratte LALT ausgebildet. NALT, Tonsillen und LALT haben unterschiedliche Altersgipfel in ihrer Größe und Funktionalität. Aufgrund dieser Unterschiede dürfen Daten von Nagerspezies nur sehr vorsichtig auf den Menschen übertragen werden. Der Begriff gemeinsames Schleimhautimmunsystem sollte durch den Begriff ,integriertes“ MALT ersetzt und die immunologischen Unterschiede zwischen Atem- und Verdauungstrakt stets berücksichtigt werden.

\section{Abstract \\ $\nabla$}

Nose- and larynx associated lymphatic tissues (NALT and LALT) vary markedly between humans, rats and mice. NALT of rats and mice is formed by paired lymphoid aggregates in the nasal cavity, while it consists of individual mucosa associated lymphoid follicles throughout the nose in humans. In addition to NALT, tonsils are present in humans, but not in rats and mice. In the larynx, LALT can be found in humans, but not in rats. Size and functionality of NALT, tonsils and LALT vary with age. The extrapolation of data obtained from rodents to humans should be carefully evaluated due to these differences. The term common mucosal immune system should replaced by the term „integrated“ MALT and the immunological differences between respiratory and digestive tract should always be considered.

\section{Nase-assoziiertes lymphatisches Gewebe \\ $\nabla$}

Die intranasale Route zur Induktion von protektiven Impfungen ist eine interessante Variante mit wenig immunpathologischen Reaktionen [1]. Deshalb besteht großes Interesse, die Lokalisation der Antigenaufnahme und Antigenverarbeitung in der Nase besser zu verstehen. Bei Ratte und Maus befinden sich paarige Ansammlungen lymphatischen Gewebes am dorsalen Boden der Nasenhöhle mit für die Antigenaufnahme spezialisierten M-Zellen, einer follikulären Ansammlung von B-Lymphozyten und in der Peripherie T-Lymphozyten (Übersicht bei [2]). Diese Struktur wird nose-associated lymphoid tissue (NALT) genannt. Cesta [3] hat die Lage des NALT und die strukturellen Elemente vom NALT bei der Ratte zusammengefasst und mit typischen Bildern belegt. Es muss festgehalten werden, dass Maus und Ratte nur diese lymphatischen Organe nach der Geburt entwickeln, aber keine Tonsillen besitzen wie der Mensch mit Tonsilla pharyngea, Tonsilla lingualis, Tonsilla palatina etc. Eine Übersicht über die Mukosaassoziierten lymphatischen Gewebe (MALT) und ihre Funktionen bei veterinärmedizinisch relevanten Spezies wurde kürzlich zusammengestellt [4]. Ein interessanter Vergleich zwischen NALT und Peyer'schen Platten des Dünndarms findet sich bei Heritage et al. [5]. Im NALT der Maus sind alle hochendothelialen Venulen (HEV) positiv für das für den peripheren Lymphknoten typische Adhäsionsmolekül des Darms (PNAd), aber ungewöhnlicherweise waren die HEV z.T. gleichzeitig positiv für das typische Adhäsionsmolekül MAdCAM-1 [6]. Beim Menschen findet man zusätzlich zu den unterschiedlichen Tonsillen auch in der Nase des Kindes Aggregate von Lymphozyten mit einer typischen Kompartimentierung. Diese NALT-Struktur befindet sich aber nicht nur am dorsalen Rachenboden wie bei Maus und Ratte, sondern ebenfalls auf den drei Conchien, dem Septum und Gaumen [7]. Es ist noch unklar, ob sich diese Ansammlungen in ihrer Funktion je nach Lokalisation unterscheiden und wie häufig sich solche Strukturen in der gesunden Nase des Erwachsenen sowie z.B. bei chronisch allergischen Reaktionen finden. Ein wesentlicher Unterschied zwischen den Nagern Maus sowie Ratte und dem Menschen ist demnach, dass NALT bei den Nagern das einzige strukturierte Gewebe im Nasen-Rachenraum ist, während NALT beim Menschen in Ergänzung zu den unterschiedlichen Tonsillen existiert, die alle unterschiedliche Altersgipfel in ihrer Größe und Funktionalität haben. Versuche an Nagern dürfen deshalb nur mit Vorsicht auf den Menschen übertragen werden.

\section{Kehlkopf (Larynx)-assoziiertes lymphatisches Gewebe \\ $\nabla$}

Die Lokalisation von Immunzellen in der Kehlkopfschleimhaut ist bei der Maus wenig, bei der Ratte aber intensiv untersucht worden. Akkumulationen von lymphatischen Zellen im Sinne eines Larynx-assoziierten lymphatischen Gewebes (LALT) kommen normalerweise bei der Ratte nicht vor. Es finden sich deutliche Unterschiede in der Häufigkeit von Granulozyten, Makrophagen, natürlichen Killerzellen und T- und B-Lymphozyten in der Region oberhalb der Stimmritze - (supraglottisch), auf dem Stimmband und kaudal vom Stimmband - (infraglottisch) [8]. Noch deutlicher war der Einstrom von dendritischen Zellen im subglottischen Raum nach einer Infektion mit Sendai Virus [9]. Ebenfalls für jede Zellart sehr unterschiedlich war die Kinetik vom Einstrom vor allem im subglottischen Raum nach einer Infektion der Ratten mit Moraxella catarrhalis oder Bordetella pertussis [10].

In der Epiglottis des Menschen fanden sich dagegen Aggregate von Lymphozyten mit typischen T- und B-Lymphozyten und HEV in den ersten zwei Lebensjahren $[11,12]$. Es bestanden keine Unterschiede in der Anzahl und Größe von LALT zwischen Kindern, die an plötzlichem Kindstod oder aus anderen Gründen gestorben waren [13]. Bei Erwachsenen nahm die Häufigkeit von LALT mit dem Lebensalter ab [14].

\section{Schlussfolgerungen \\ $\nabla$}

Es bestehen deutliche Speziesunterschiede zwischen Nagern (Maus, Ratte) und dem Menschen sowie ausgeprägte Altersunterschiede. Deshalb dürfen nur sehr vorsichtig Daten von diesen Nagerspezies auf den Menschen übertragen werden. Auch aus 
diesen Gründen sollte der Begriff gemeinsames Schleimhautimmungewebe (common mucosa-associated lymphoid tissue) durch den Terminus „intergriertes“ MALT ersetzt [15] und die immunologischen Unterschiede zwischen Atem- und Verdauungstrakt stets berücksichtigt werden [16].

\section{Literatur}

1 Hussell T, Humphreys IR. Nasal vaccination induces protective immunity without immunopathology. Clin Exp Immunol 2002; 130: 359-362

2 Kraal G. Nasal-asociated lymphoid tissue. Mucosal Immunology, Third Edition. I. In: Mestecky J, Lamm ME, Strober W et al., eds. Amsterdam: Academic Press; 2005: 415-422

3 Cesta MF. Normal structure, function, and histology of mucosa-associated lymphoid tissue. Tox Path 2006; 34: 599-608

4 Liebler-Tenorio EM, Pabst R. MALT structure and function in farm animals. Vet Res 2006; 37: 257-280

5 Heritage PL, Underdown BJ, Arsenault AL et al. Comparison of murine nasal-associated lymphoid tissue and Peyer's Patches. Am J Respir Crit Care Med 1997; 156: 1256-1262

6 Csencsits KL, Jutila MA, Pascual DW. Nasal-associated lymphoid tissue: Phenotypic and functional evidence for the primary role of peripheral node adressin in naive lymphocyte adhesion to high endothelial venules in a mucosal site. J Immunol 1999; 0: 1383-1389

7 Debertin AS, Tschernig T, Tönjes $H$ et al. Nasal-associated lymphoid tissue (NALT): frequency and localization in young children. Clin Exp Immunol 2003; 134: 503-507

8 Jecker P, Ptok M, Pabst R, Westermann J. Distribution of immunocompetent cells in various areas in the normal larygeal mucosa of the rat. Eur Arch Otorhinolaryngol 1996; 253: $142-146$

9 Jecker P, McWilliam A, Marsh A et al. Acute Laryngotracheitis in the rat induced by Sendai virus: the influx of six different types of immunocompetent cells into the laryngeal mucosa differs strongly between the subglottic and the glottic compartment. Laryngoscope 2001; 111: $1645-1651$

10 Jecker P, McWilliams A, Napoli S et al. Acute laryngitis in the rat induced by Moraxella catarrhalis and Bordetella pertussis : number of Neutrophils, dendritic cells, and $\mathrm{T}$ and $\mathrm{B}$ lymphocytes accumulating during infection in the laryngeal mucosa strongly differs in adjacent locations. Pedriat Res 1999; 46, 6: 760-766

11 Kracke A, Hiller AS, Tschernig TR et al. Larynx-associated lymphoid tissue (LALT) in young children. The Anat Rec 1997; 248: 413-420

12 Kracke A, Hiller AS, Kasper $M$ et al. The larynx of young children contains mucosa-associated lymphoid tissue. Amsterdam: Elsevier Science B.V.; 1997: 364- 368

13 Hiller AS, Kracke A, Tschernig T et al. Comparison of the immunohistology of mucosa-associated lymphoid tissue in the larynx and lungs in cases of sudden infant death and controls. Int J Legal Med 1997; 110: $316-322$

14 Hiller AS, Tschernig T, Kleemann WJ, Pabst R. Bronchus-associated lymphoid tissue (BALT) and larynx-associated lymphoid tissue (LALT) are found at different frequencies in children, adolescents and adults. Scand J Immunol 1998; 47: 159-162

15 Brandtzaeg P, Pabst R. Let's go mucosal: communication on slippery ground. Trends Immunol 2004; 25: 570-577

16 Kunisawa J, Nochi T, Kiyono H. Immunological commonalities and distinctions between airway and digestive immunity. Trends Immunol 2008; 29: 505-513

Bibliografie

DOI $10.1055 / \mathrm{s}-0030-1255509$

Pneumologie 2010; 64; 443-455

(c) Georg Thieme Verlag KG Stuttgart · New York ISSN 0934-8387

\section{Korrespondenzadresse}

Prof. Dr. Reinhard Pabst

Institut für Immunmorphologie 4160

Medizinische Hochschule Hannover

Carl-Neuberg-Str. 1

30625 Hannover

pabst.reinhard@mh-hannover.de

\section{Prinzipielle Unterschiede am Stimmapparat von Mensch und Schwein: Einige wesentliche morphologische Kriterien}

\author{
R. Koch ${ }^{1}$, I. Hennig-Pauka ${ }^{2}$, H. Gasse ${ }^{1}$ \\ 1 Anatomisches Institut, Tierärztliche Hochschule Hannover \\ 2 Klinik für Kleine Klauentiere, Tierärztliche Hochschule Hannover
}

\section{Zusammenfassung}

$\nabla$

In der Glottis lässt der Feinbau der Lamina propria beim Schwein - vor allem an der kranialen Falte - andere phoniatrische Eigenschaften als beim Menschen erwarten. Beim Speziesvergleich müssen altersabhängige Entwicklungen berücksichtigt werden.

\section{Abstract}

The histological composition of the Lamina propria in pigs especially in the cranial fold of the glottis - gives reason to expect other phoniatric properties than in man. Age-related changes must be considered.

Der Schweinekehlkopf findet als Modell für den Kehlkopf des Menschen zunehmend Verwendung [1,2]; er soll unter diversen Spezies im Hinblick auf die Phonation dem des Menschen am ehesten entsprechen [3]. Vergleichende Studien befassen sich insbesondere mit dem Bau der Lamina propria der Stimmfalte (Plica vocalis) [4-6]. Hierbei wird beim Schwein immer nur die kaudal des Ventriculus laryngis gelegene Falte untersucht.

Die kranial des Zugangs gelegene Falte gleicht nicht der beim Menschen hier gelegenen Taschenfalte (Plica vestibularis, auch Plica ventricularis genannt). Die Taschenfalte (Mensch) ragt nicht sehr weit ins Lumen vor und ist nicht schwingungsfähig. Sie wird von einer lockermaschigen Respirationsschleimhaut mit zahlreichen seromukösen Drüsen gebildet. Quergestreifte Muskulatur und ein kräftiger Bandapparat fehlen [7]. Die kraniale Schleimhautfalte des Schweins dagegen ist mit einem mehrschichtigen Plattenepithel [8] bedeckt und enthält dicke Kollagenfaserbündel: sie entspringen gemeinsam mit jenen der kaudalen „wahren" Stimmfalte am Aryknorpel und inserieren am Schildknorpel [9]. Dem Bau und Verlauf gemäß stellt dies also eine kraniale Stimmfalte dar. So sprechen auch Alipour und Jaiswal (2009) von einer oberen und unteren Stimmfalte; die obere (kraniale) Stimmfalte ist stärker gespannt und erwies sich bei Schwingungsversuchen als Haupt-Oszillator [10].

Die Lamina propria der Stimmfalte des Menschen wird histologisch in drei Schichten eingeteilt: superficial, intemediate, deep layer of lamina propria (SLLP, ILLP, DLLP). Die SLLP (Reinke-Raum) besteht aus lockerem Bindegewebe; in der ILLP überwiegen elastische, in der DLLP Kollagenfasern [10]. ILLP und DLLP bilden das Ligamentum vocale, den verstärkten Rand des Conus elasticus. Hahn et al. (2005) [12] unterscheiden zusätzlich die subepitheliale Basalmembran-Zone (BMZ). Diese Schichten haben essentielle Bedeutung für die Phonation (Body-Cover-Modell, $[13,14])$. 
Die Lamina propria der (kaudalen) Stimmfalte des Schweins wird stattdessen als zweischichtig beschrieben, allerdings ohne eindeutige Grenze zwischen beiden. Die oberflächliche Schicht besteht überwiegend aus Grundsubstanz, die tiefe vorwiegend aus Kollagen und minimal Elastin [1]. Daher verneinen einige Autoren die Existenz eines Conus elasticus und eines elastischen Stimmbandes beim Schwein [4] und vergleichen sie mit der Stimmfalte eines Kindes [2].

Der Mensch besitzt bei der Geburt noch kein Stimmband, die gesamte Lamina propria besteht aus lockerem, grundsubstanzreichen Bindegewebe; die vollständige Ausbildung der Schichten der Lamina propria erfolgt geschlechtsabhängig erst im Alter von ca. 17 Jahren [15]. Entsprechende Angaben zum Schwein fehlen in der Literatur; mit Blick auf dieses wichtige struktur- und funktionsrelevante Kriterium untersuchten wir darum die kraniale und die kaudale Falte des Stimmapparates von weiblichen Schweinen, die 14 Tage, 6 Monate oder 5 Jahre alt waren.

Erste - aufgrund geringer Stichprobenzahl - vorläufige Befunde sind folgende:

1. Die kaudale Falte des Stimmapparats vom Schwein gleicht histologisch nicht der Plica vocalis des Menschen; eine 3Schichtigkeit als Organisationsmuster ist beim 6 Monate alten Schwein nur vage (fließende Übergänge), beim 5 Jahre alten (durchgängig kollagenfaserreich) gar nicht erkennbar. Generell fällt bei beiden die Durchsetzung der Schichten mit elastischen Fasern auf; beim letztgenannten tritt eine starke Häufung auf, allerdings nicht als „Schicht“, sondern als Konzentrierung im kranialen Bereich der Falte. Schon beim 14 Tage alten Schwein kommen elastische Fasern vor, insbesondere in der DLLP, mehr noch in der kollagenen und elastischen BMZ.

2. Die kraniale Falte des Stimmapparats hat eine sehr dicke Lamina propria. Schon beim 14 Tage alten Schwein zeichnen sich anhand der Kollagenfasern deutlich BMZ, SLLP, ILLP und DLLP ab; die Dicke der DLLP macht einen deutlichen Unterschied zur Plica vocalis des Menschen aus. Elastische Fasern treten gehäuft in der ILLP auf, und ganz besonders in der BMZ; sie reichen bei der Gewebeprobe vom 5 Jahre alten Schwein tief in die subepithelialen Zapfen des Papillarkörpers. Auch insofern liegt ein deutlicher Unterschied zur Plica vocalis des Menschen vor.

\section{Literatur}

1 Garrett CG, Coleman JR, Reinisch L. Comparative histology and vibration of the vocal folds: implications for experimental studies in microlaryngeal surgery. Laryngoscope 2000; 110: 814-824

2 Blakeslee DB, Banks RE, Eusterman Vet al. Analysis of vocal fold function in the miniswine model. J Invest Surg 1995; 8: 409-424

3 Jiang JJ, Raviv JR, Hanson DG. Comparison of the phonation-related structures among pig, dog, white-tailed deer, and human larynges. Ann Otol Rhinol Laryngol 2001; 110: 1120-1125

4 Kurita S, Nagata K, Hirano M. A comparative study of the layer structure of the vocal fold.In: Bless DM, Abbs JH, Hrsg Vocal fold physiology. San Diego: College-Hill Press; 1983: 3-21

5 Hahn MS, Kobler JB, Starcher BC et al. Quantitative and comparative studies of the vocal fold extracellular matrix. I. Elastic fibers and hyaluronic acid. Ann Otol Rhinol Laryngol 2006; 115: 156-164

6 Hahn MS, Kobler JB, Zeitels SM et al. Quantitative and comparative studies of the vocal fold extracellular matrix. II. Collagen. Ann Otol Rhinol Laryngol 2006; 115: 225-232

7 Rohen JW, Lütjen-Drecoll E. Funktionelle Histologie. Stuttgart, New York: Schattauer; 2000

8 Burow W. Anatomie und Histologie des Kehlkopfes einiger Haussäugethiere. Diss. Univ Zürich: 1902

9 Waibl H. Atmungsapparat, Apparatus respiratorius. In: Frewein J, Gasse H, Leiser R et al., Hrsg. Lehrbuch der Anatomie der Haustiere. Bd. II Eingeweide. Berlin: Parey; 1999: 223-307
10 Alipour F, Jaiswal S. Glottal airflow resistance in excised pig, sheep, and cow larynges. J voice 2009; 23: 40-50

11 Hirano M, Kurita S, Nakashima T. Growth, development and aging of human vocal folds.In: Bless DM, Abbs JH, Hrsg Vocal fold physiology. San Diego: College-Hill Press; 1983: 22-43

12 Hahn MS, Kobler JB, Zeitels SM et al. Midmembranous vocal fold lamina propria proteoglycans across selected species. Ann Otol Rhinol Laryngol 2005; 114: $451-462$

13 Nawka T, Hoseman W. Gestörte Stimme. Chirurgische Verfahren. Laryngo-Rhino-Otol 2005; 84: 201 - 215

14 Gray SD, Titze IR, Alipour F et al. Biomechanical and histologic observations of vocal fold fibrous proteins. Ann Otol Laryngol 2000; 109: 77 85

15 Ishii K, Yamashita K, Akita $M$ et al. Age-related development of the arrangement of connective tissue fibers in the lamina propria of the human vocal fold. Ann Otol Laryngol 2000; 109: 1055-1064

\section{Bibliografie}

DOI $10.1055 / \mathrm{s}-0030-1255510$

Pneumologie 2010; 64; 443-455

(c) Georg Thieme Verlag KG Stuttgart · New York

ISSN 0934-8387

Korrespondenzadresse

Dr. R. Koch

Anatomisches Institut,

Tierärztliche Hochschule Hannover

Bischofsholer Damm 15

30173 Hannover

Ruediger.Koch@tiho-hannover.de

\section{Obstruktion der oberen Atemyege Obstruktion der oberen Atemwege beim Menschen und Tiermodelle}

\section{R. Schulz}

Universitätsklinik Gießen und Marburg, Medizinische Klinik II, Schlafmedizinisches Labor

\section{Zusammenfassung}

Die obstruktive Schlaf-Apnoe (OSA) wird durch den repetitiven Kollaps der oberen Atemwege im Schlaf verursacht, wobei Adipositas einen der Hauptrisikofaktoren darstellt. Die im Schlaf auftretenden Atempausen führen zunächst zum Abfall des Sauerstoff- und Anstieg des Kohlendioxidpartialdruckes, in der Folge zur Sympathikusaktivierung (Anstieg von Blutdruck und Herzfrequenz) sowie zu frustranen Atemanstrengungen und schließlich zur zentralnervösen Weckreaktion (=Arousal). Obwohl kaum ein Tiermodell in der Lage ist, diese Komplexität der pathophysiologischen Reaktionen widerzuspiegeln, wurde die Grundlagenforschung der OSA durch den Einsatz von Tiermodellen bzw. Tierexperimenten sehr bereichert. Das zukünftige Ziel muss es sein, die gewonnenen Erkenntnisse möglichst nutzbringend wieder auf OSA-Patienten zu übertragen. In diesem Zusammenhang ist z. B. an die Pharmakotherapie des pharyngealen Kollaps oder eine antioxidativ ausgerichtete Therapie der kardiovaskulären u./o. ZNS-Auswirkungen der OSA zu denken. Insbesondere OSA-Patienten, die eine CPAP-Therapie nicht tolerieren, könnten hiervon profitieren. 


\section{Abstract}

$\nabla$

Obstructive sleep apnea (OSA) is caused by repetitive collapse of a narrow upper airway during sleep with the main risk factor being obesity. Apneas are followed by hypoxia, sympathetic activation, intrathoracic pressure swings and arousals. In most animal studies, only the cyclical pattern of hypoxia characteristic of OSA is simulated, however, more complex models have also been developed which additionally reflect the other pathophysiological changes associated with sleep-disordered breathing. These models have contributed to a deeper understanding of the cardiovascular and metabolic consequences of OSA. From other experiments the concept of the pharynx behaving like a collapsible tube, i.e. a Starling resistor, has emerged. Finally, the neurotransmitter modulation of upper airway muscle tone has been elucidated by using in vivo microdialysis of the caudal medulla of rats. It is hoped that findings from animal studies will in the future impact on the management of patients with OSA, in particular if they are non-compliant with CPAP therapy.

\section{Obstruktive Schlaf-Apnoe beim Menschen \\ $\nabla$}

Die obstruktive Schlaf-Apnoe (OSA) wird durch den repetitiven Kollaps der oberen Atemwege im Schlaf verursacht. Hauptrisikofaktor ist die Adipositas, die durch eine Vermehrung der lateralen Fettpolster zu einer Pharynx-Einengung führt [1]. Bei nichtadipösen OSA-Patienten kann dies durch skelettale Faktoren wie z. B. eine Retrognathie bedingt sein [2]. Weiterhin spielt bei dieser Subgruppe offenbar ein „fluid shift“ im Liegen von der unteren in die obere Körperhälfte eine Rolle [3]. Begünstigt wird der pharyngeale Kollaps schließlich durch einen Verlust der neuromuskulären Kompensation im Schlaf, d.h. durch eine Tonusabnahme der den Pharynx dilatierenden Muskeln, insbesondere des M. genioglossus [4].

Der Pharynx-Kollaps hat einen Abfall des Sauerstoffpartialdruckes und einen Anstieg des Kohlendioxidpartialdruckes zur Folge. Durch diese Blutgasveränderungen resultiert eine Sympathikusaktivierung, was wiederum einen Anstieg von Blutdruck und Herzfrequenz bewirkt.

Weiterhin finden sich bei der OSA während der Atempausen frustrane Atemanstrengungen, die zu einer Negativierung des intrathorakalen Drucks führen. Die genannten Veränderungen münden schließlich in eine zentralnervöse Weckreaktion (Arousal), welche die Atempause durch Wiedereröffnung des Pharynx terminiert. Während der sich anschließenden Hyperventilation erfahren die erwähnten physiologischen Parameter gegenläufige Veränderungen, z. B. Wiederanstieg der $\mathrm{O}_{2}$-Sättigung usw. Durch die wiederholte Abfolge von Apnoen und Hyperventilationsphasen sind bei der OSA letztendlich zyklische Schwankungen aller Messgrößen vorhanden, z. B. intermittierende Hypoxie.

\section{Tiermodelle}

Es existieren verschiedene Tiermodelle der OSA, wobei im Wesentlichen eine Unterteilung in 3 Gruppen erfolgen kann. Natürlich vorkommende Modelle sind z.B. die englische Bulldoge und das adipöse Minischwein, die aber beide nur begrenzt in der tierexperimentellen Forschung zur OSA zum Einsatz kommen [5,6]. Am weitesten verbreitet ist das Modell der chronisch-intermittierenden Hypoxie ( $\mathrm{CIH}$ ), in welchem die Tiere (meistens Ratten oder Mäuse) in Versuchskammern gehalten werden, in denen das Muster der OSA-assoziierten Hypoxie durch automatisiertes Ein- und Auspumpen definierter Gasgemische simuliert wird [7]. Nachteil dieses Modells ist, dass es andere pathophysiologische Veränderungen im Rahmen der OSA wie z.B. intrathorakale Druckschwankungen nicht abbildet. Dies wird erst im Rahmen komplexer, instrumentierter Modelle möglich, in denen der repetitive Kollaps der oberen Atemwege nachvollzogen wird. Hierzu kann z.B. ein wechselweises Schließen und Öffnen einer im Atemstrom befindlichen Klappe eingesetzt werden [8,9].

Die genannten Tiermodelle haben zu einem besseren Verständnis der Pathophysiologie der Folgeerkrankungen der OSA beigetragen. Dies betrifft vor allem die Auswirkungen der OSA auf kardiovaskuläre und metabolische Parameter, aber auch das zentrale Nervensystem. Bezüglich tierexperimenteller Untersuchungen zu den kardiovaskulären Komplikationen der OSA sei an dieser Stelle auf eine frühere Übersichtsarbeit verwiesen [10]. Studien zu den metabolischen Auswirkungen der OSA haben gezeigt, dass es bei Ratten und Mäusen unter $\mathrm{CIH}$ zur Entwicklung von Insulinresistenz, Hyperlipidämie und Fettleber kommt [11]. Ob die hierbei identifizierten Pathomechanismen auch für Patienten mit OSA zutreffen, muss allerdings noch geklärt werden. Es konnte auch gezeigt werden, dass die $\mathrm{CIH}$ bei Versuchstieren zu neurokognitiver Dysfunktion und Müdigkeit führt.

In bestimmten Hirnarealen von Mäusen wurde eine Hochregulation des $\mathrm{O}_{2}$-Radikalen produzierenden Enzyms NADPH-Oxidase gefunden, so dass die ZNS-Funktionsveränderungen bei der OSA ähnlich wie bereits die kardiovaskulären Folgeerkrankungen in Verbindung mit einem erhöhten oxidativen Stress gebracht wurden [12].

Im weiteren Sinne haben Tierexperimente auch das Verständnis der Pathophysiologie der oberen Atemwege bei der OSA erweitert. So wurde das Konzept aufgestellt, dass die oberen Atemwege als Starling-Resistor aufzufassen sind, in dem es bei Unterschreitung des sogenannten kritischen Verschlussdrucks (= Pcrit) zum pharyngealen Kollaps kommt. Hierzu wurden die oberen Atemwege von Ratten u./o. Mäusen „isoliert“, d.h. es wurde ein trachealer Bypass angelegt und Druck - bzw. Flussmessungen ober - und unterhalb des kollapsiblen Pharynxsegmentes durchgeführt [13].

Weiterhin wurde die Neurotransmitter-Modulation der pharyngealen Muskulatur tierexperimentell aufgeklärt. Über einen in die kaudale Medulla oblongata von Ratten implantierten Mikrodialyse-Katheter wurden verschiedene chemische Substanzen appliziert und gleichzeitig die Muskelaktivität im Pharynx gemessen. Diese Studien ergaben, dass vor allem Serotonin den N. hypoglossus stimuliert und dann via Kontraktion des M. genioglossus eine Dilatation des Pharynx bewirkt [14]. Versuche, diese Erkenntnisse therapeutisch zu nutzen, sind bisher allerdings leider erfolglos gewesen [15].

\section{Literatur}

1 Schwab RJ, Pasirstein M, Pierson R et al. Identification of upper airway anatomic risk factors for obstructive sleep apnea with volumetric magnetic resonance imaging. Am J Respir Crit Care Med 2003; 168: 522 530

2 White DP. Pathogenesis of obstructive and central sleep apnea. Am J Respir Crit Care Med 2005; 172: 1363-1370

3 Redolfi S, Yumino D, Ruttanaumpawan $P$ et al. Relationship between overnight rostral fluid shift and obstructive sleep apnea in nonobese men. Am J Respir Crit Care Med 2009; 179: 241 - 246

$4 \mathrm{Katz}$ ES, White DP. Genioglossus activity during sleep in normal control subjects and children with obstructive sleep apnea. Am J Respir Crit Care Med 2004; 170: 553 - 560 
5 Hendricks JC, Kline LR, Kovalski RJ et al. The English bulldog: a natural model of sleep-disordered breathing. J Appl Physiol 1987; 63: 13441350

6 Lonergan RP, Ware JC, Atkinson RL et al. Sleep apnea in obese miniature pigs. J Appl Physiol 1998; 84: 531 - 536

7 Fletcher EC. Physiological consequences of intermittent hypoxia: systemic blood pressure. J Appl Physiol 2001; 90: 1600-1605

8 Brooks D, Horner RL, Kozar LF et al. Obstructive sleep apnea as a cause of systemic hypertension. Evidence from a canine model. J Clin Invest 1997; 99: 106-109

9 Farré $R$, Nácher M, Serrano-Mollar A et al. Rat model of chronic recurrent airway obstructions to study the sleep apnea syndrome. Sleep 2007; 30: 930-933

10 Schulz R, Eisele HJ, Murzabekova G, Weissmann N. Schlaf-Apnoe und Herzkreislauf-Erkrankungen: Ergebnisse tierexperimenteller Untersuchungen. Pneumologie 2008; 62: 18-22

11 Jun J, Polotsky VY. Sleep-disordered breathing and metabolic effects: evidence from animal models. Sleep Med Clin 2007; 2: 263-277

12 Zhan G, Serrano F, Fenik P et al. NADPH oxidase mediates hypersomnolence and brain oxidative injury in a murine model of sleep apnea. Am J Respir Crit Care Med 2005; 172: 921 - 929

13 Liu A, Pichard L, Schneider $H$ et al. Neuromechanical control of the isolated upper airway of mice. J Appl Physiol 2008; 105: 1237-1245

14 Horner RL. Respiratory motor activity: influence of neuromodulators and implications for sleep-disordered breathing. Can J Physiol Pharmacol 2007; 85: 155 - 165

15 Veasey SC. Serotonin agonists and antagonists in obstructive sleep apnea: therapeutic potential. Am J Respir Med 2003; 2: 21 - 29

\section{Bibliografie}

DOI 10.1055/s-0030-1255511

Pneumologie 2010; 64; 443-455

(c) Georg Thieme Verlag KG Stuttgart · New York

ISSN 0934-8387

\section{Korrespondenzadresse}

Prof. Dr. Richard Schulz

Universitätsklinik Gießen und Marburg,

Medizinische Klinik II

Schlafmedizinisches Labor

Paul-Meimberg-Str. 5

35392 Gießen

Richard.Schulz@innere.med.uni-giessen.de

\section{Upper airway resistance: species-related differences}

\author{
N. Kirschvink ${ }^{1}$, P. Reinhold ${ }^{2}$ \\ ${ }^{1}$ Animal Physiology, Veterinary Department, Faculty of Sciences, \\ University of Namur \\ 2 Institute of Molecular Pathogenesis in the „Friedrich-Loeffler- \\ Institut“ (Federal Research Institute for Animal Health)
}

\section{Zusammenfassung}

Den Strömungswiderständen der oberen Atemwege wird in der Tiermedizin hauptsächlich beim Sportpferd sowei beim brachyzephalen Hund eine große Bedeutung beigemessen. Aufgrund anatomischer Besonderheiten des Nasen- und Rachenraums und/oder pathologischer Veränderungen können bei beiden Spezies signifikante Veränderungen der Leistungstoleranz (Performance) und/oder des Wohlbefindens auftreten. Bei anderen Tierarten wird den physiologischen Eigenschaften und den patholo- gischen Veränderungen der unteren Atemwege meist eine größere Bedeutung beigemessen.

\section{Abstract \\ $\nabla$}

In veterinary medicine, upper airway resistance deserves a particular attention in equines athletes and brachycephalic dogs. Due to the anatomical peculiarities of the upper airway and/or pathological conditions, significant alterations of performance and/or well being might occur in horses and dogs. Physiological specificities and pathological changes of the lower respiratory tract deserve a major attention in other species.

\section{Introduction \\ $\nabla$}

The measurement of lung function and determination of lower and upper airway resistances in animal species was first performed in the equine species. Horses are obligate nasal breathers and their respiratory system is the performance limiting system (even in healthy conditions!). Indeed, the equine athlete naturally develops exercise-induced hypoxemia, which seems to be related to an insufficient oxygen transfer throughout the capillary-alveolar barrier as well as to inappropriate ventilation due to strongly increased airflow resistance (more than $100 \%$ increase) [1]. The investigation methods developed for equines have been extended to other animal species where the main reasons for measuring upper and lower airway resistance are investigation of pathophysiology and therapy of respiratory diseases. As certain animal peculiarities might model respiratory disorders in man, there is some interest for animals' upper airway in human medicine.

\section{Measurement techniques of upper airway resistance in animals: conventional pulmonary function tests and impulse oscillometry}

The first measurements of upper and lower airway resistance (respectively UAR and LAR) were performed by use of so called conventional pulmonary function tests where transpulmonary, tracheal and naso-pharyngeal pressures and airflow need to be recorded [2]. Impulse oscillometry has also been validated for several species in respiratory veterinary research and appears as a more sensitive and less invasive technique: a spectrum of frequencies is taken into consideration and calculation models of upper and lower (or central and peripheral) airway resistance are available [3].

\section{Upper airway resistance in animal species $\nabla$}

As a consequence of anatomical peculiarities of their upper airways, horses show the highest UAR:LAR ratio and rest and at exercise ( Table 1 ), associated with a considerable work of breathing [2].

The functional impact of several affections of UA, i.e. laryngeal paresis or paralysis, soft dorsal palate displacement, guttural pouch mycosis or empyema etc. can be quantified by use of an impulse oscillometry system (IOS) [4] or measurement of pressure changes [5]. Although the IOS technique offers several advantages in terms of sensitivity and non-invasiveness, it can not be used in exercising horses.

Cattle, and especially hypermuscled breeds such as Belgian White Blue, slowly adapt their respiratory function after birth. Their 
Table 1 Ratio between upper airway resistance (UAR) and lower airway resistance (LAR) in animal species.

\begin{tabular}{ll} 
Animal species & $\begin{array}{l}\text { UAR: LAR ratio (\%) in normal } \\
\text { conditions (mature animals) }\end{array}$ \\
& $80: 20$ \\
\hline Horse & $70: 30$ \\
\hline Cattle & $70: 30$ \\
\hline Sheep & $60: 40$ \\
\hline
\end{tabular}

\begin{tabular}{ll}
$\begin{array}{l}\text { Ratio change in particular } \\
\text { conditions }\end{array}$ & References \\
Exercise $82: 18$ & {$[1,2]$} \\
\hline Young animals $60: 40$ & {$[7,8]$} \\
\hline- & Reinhold; unpublished \\
\hline Young animals 55:45 & {$[9]$}
\end{tabular}

anatomical and physiological peculiarities of the respiratory system, i.e. a relatively small respiratory tract, an important development of pleural septa, the absence of collateral ventilation, and an important capacity of hypoxic vasoconstriction, explain why this species is prone to respiratory affections of the upper and lower airways [6]. Comparative assessment of respiratory function between growing Friesian and BWB calves has further provided evidence that the UAR:LAR ratio decreases with age due to decreased LAR and that both, UAR and LAR are higher in BWB $($ Table 1) $[7,8]$.

Similar growth-related changes are described in pigs; the relative impact of lower airway resistance remains however higher than in other species ( $\bullet$ Table 1 ) [9].

Although cats have been used during early respiratory research for investigating nervous control of respiration, upper airways have been poorly investigated from a clinical point of view. In dogs, especially brachycephalic dogs presenting considerable nasal airflow resistance, resistive properties of upper airways are under ongoing investigation [10].

\section{References}

1 Art T, Lekeux P. Work of breathing in exercising ponies. Res Vet Sciences 1989; 46: 49-53

2 Art T, Serteyn D, Lekeux P. Effect of exercise on the partitioning of equine respiratory resistance. Equine Vet J 1988; 20: 268-273

3 Smith H-J, Reinhold P, Goldman MD. Forced oscillation technique and impulse oscillometry. European Respiratory Monograph 2005; 31: $72-105$

4 Van Erck E, Votion D, Art T et al. Qualitative and quantitative evaluation of equine respiratory mechanics by impulse oscillometry. Equine Vet J 2006; 38: $52-58$

5 Rakesh V, Ducharme NG, Cheetham J et al. Implications of different degrees of arytenoid cartilage abduction on equine upper airway characteristics. Equine Vet J 2008; 40: 629-635

6 Kirschvink $N$. Respiratory function in cattle: impact of breed, heritability and external factors. Dtsch Tierarztl Wochenschr 2008; : 265-270

7 Reinhold P, Smith H-J, Close R et al. Validation of impulse oscillometry in Friesian and Blue Belgian calves with respect to changes in extrathoracic upper airway resistance. Res Vet Sciences 1998; 65: 93-102

8 Jaeger J, Liebler-Tenorio E, Kirschvink $N$ et al. A clinically silent respiratory infection with Chlamydophila spp. in calves is associated with airway obstruction and pulmonary inflammation. Vet Res 2007; 38 : $711-728$

9 Wagner J, Kneucker A, Liebler-Tenorio E et al. Respiratory function and pulmonary lesions in pigs infected with porcine reproductive and respiratory syndrome virus. Vet J 2010 [Epub ahead of print] doi: 10.1016/j.tvjl.2009.12.022

10 Hueber JP, Smith H-J, Reinhold P et al. Untersuchungen zur Geometrie und Funktion der Hundenase - Wie verändert sich die Funktion, wenn die Form verändert wird? Pneumologie 2010; 64 (Suppl.3): S254
Bibliografie

DOI $10.1055 / \mathrm{s}-0030-1255512$

Pneumologie 2010; 64; 443-455

(c) Georg Thieme Verlag KG Stuttgart · New York

ISSN 0934-8387

Korrespondenzadresse

PD Dr. Dr. Petra Reinhold

Friedrich-Loeffler-Institut

Institut für molekulare Pathogenese

Naumburger Str. 96a

07743 Jena

Germany

petra.reinhold@fli.bund.de

\section{Brachyzephalie bei Hund und Katze: eine "menschengemachte ${ }^{\text {" }}$ Obstruktion der oberen Atemwege}

\section{G. U. Oechtering, C. Schlüter, J. P. Lippert}

Klinik für Kleintiere, Veterinärmedizinische Fakultät der Universität Leipzig

\section{Zusammenfassung}

Durch übertriebene Zuchtauslese wurde bei vielen brachyzephalen Rassen die Nase nahezu „weggezüchtet“, mit erheblichen anatomischen und funktionellen Konsequenzen.

Zusätzlich zu respiratorischen und olfaktorischen Aufgaben ist die Hundenase von vitaler Bedeutung für die Thermoregulation. Hunde sind obligate Nasenatmer und leiden bei jeder Einschränkung der nasalen Ventilation wesentlich stärker als der Mensch. Eine offene Diskussion in der Gesellschaft muss das Handlungsbedürfnis von Behörden und Zuchtverbänden steigern, die Zucht auf extreme Brachyzephalie als tierschutzrelevante Form der Qualzucht zu erkennen und entsprechend zu handeln.

\section{Abstract \\ $\nabla$}

Selective breeding for exaggerated features caused in many brachycephalic dog and cat breeds virtually a loss of the nose, with serious anatomical and functional consequences.

In addition to respiratory and olfactory tasks, in dogs the nose is of vital importance for thermoregulation. As obligatory nose breathers, dogs suffer far more than humans when their nasal ventilation is restricted.

An open discussion in the broad public has to motivate authorities and kennel clubs to recognize extreme brachycephalic breeding as seriously affecting animal health and welfare. 


\section{Vorbetrachtungen \\ $\nabla$}

Treten bei der Hundezucht gebrauchsorientierte Interessen (z. B.: Jagd-, Dienst- und Hütehunde) in den Hintergrund - und nimmt damit die Notwendigkeit ab, gesunde und leistungsfähige Hunde zu züchten - so kann die Überbetonung äußerlicher Merkmale zur Einschränkung physiologischer Organfunktionen führen. Kurzköpfige Hunde- und Katzenrassen erfreuen sich wegen ihres stupsnasigen Aussehens (Kindchenschema [1]) und ihres in der Regel sehr menschenfreundlichen Wesens weltweit zunehmender Beliebtheit. Brachyzephale Rassen haben eine angeborene Wachstumshemmung des Splanchnokraniums, dadurch behalten auch adulte Tiere ein welpenartiges Aussehen. Kurzköpfige Rassen gelten als relativ alt, brachyzephale Hundeschädel wurden bereits in der Asche Pompeis gefunden [2]. In den vergangenen Jahrzehnten wurde durch übertriebene züchterische Selektion die Schädelform derart verkürzt, dass heute gravierende Atemprobleme ein hervorstechendes Merkmal dieser Rassen sind. Angestrengte Atmung in Ruhe, schnarchende Atmungsgeräusche (auch in wachem Zustand!), Schlafapnoe und ausgeprägte Belastungs- und Wärmeintoleranz sowie eine Vielzahl weiterer, nicht-respiratorischer Probleme gelten als klassische Symptome. Damit sind im Tierschutzgesetz definierte Kriterien der Qualzucht klar erfüllt.

\section{Anatomische Betrachtungen und neuere Erkenntnisse $\nabla$}

Bisher wurden als typische patho-anatomische Veränderungen eine Trias aus stenotischen Nares, überlangem Palatum molle und evertierten seitlichen Kehlkopftaschen beschrieben [3]. Wir haben die bisher nicht beschriebenen Auswirkungen eines dramatisch reduzierten „Mittelgesichtes“ auf die nasalen Binnenstrukturen untersucht. Hierfür wurden über dreihundert zur Diagnostik und Behandlung schwerer respiratorischer Beschwerden überwiesene brachyzephale Hunde und Katzen einbezogen. Die oberen Atemwege wurden computertomografisch und endoskopisch dargestellt; Hauptaugenmerk lag auf Struktur und Ausdehnung der Nasenmuscheln. Kraniometrische Untersuchungen sollten das Verhältnis von Splanchnokranium zu Neurokranium beschreiben [4], rhinometrische Untersuchungen die Relevanz von computertomografisch oder endoskopisch festgestellten Stenosen verdeutlichen [5]. Pathohistologische Untersuchungen von physiologischen und brachyzephalen Nasenmuscheln sollten Aufschluss über die Veränderungen in der Feinstruktur geben [6]. Die Ergebnisse unserer Untersuchungen offenbarten, dass die extreme Verkürzung des Splanchnokraniums zu dramatischen Veränderungen aller nasalen Binnenstrukturen geführt hat $[4,7]$, was folgende strukturelle und funktionelle Konsequenzen nach sich zieht:

- Nares und Vestibulum: Anders als beim Menschen ist der Nasenvorhof beim Hund nicht leer. Er wird ausgefüllt von einem voluminösen, nach kaudal ziehenden Nasenflügel, der in die Flügelfalte übergeht. Die Stenose des Naseneingangs von brachyzephalen Hunden ist wesentlich komplexer als bisher angenommen. An eine von außen gut sichtbare Stenose der Nares schließt sich eine nur endoskopisch darstellbare ausgeprägte Stenose des Vestibulums an.

- Nasenhöhle: Verkleinert man durch Zuchtauslese den knöchernen Rahmen eines Organs auf weniger als ein Drittel der natürlichen Größe ( $\bullet$ Abb. 1), muss dies für die darin enthaltenen Strukturen erhebliche Konsequenzen haben. Im Gegensatz zu den relativ einfach aufgebauten Nasenmuscheln des Menschen, haben Hunde und Katzen hochkomplexe, extrem fein verzweigte Konchenstrukturen. Bei Brachyzephalen sind die Nasenmuscheln im Verhältnis zur umgebenden knöchernen Begrenzung wesentlich zu groß und wir sprechen von einer relativen Konchenhypertrophie. Sie führt dazu, dass die feinen Lamellen der Nasenmuscheln bis zum Kontakt miteinander wachsen und kaum noch Raum für die durchströmende Luft bleibt. Zusätzlich wachsen Muschelanteile als aberrante Conchen in Atemwege hinein und verlegen diese. Man kann zwischen „rostralen aberranten Conchen“ (RAC) und „kaudalen aberranten Conchen“ (CAC) differenzieren [8]. Auch die Feinstruktur der Conchen ist bei brachyzephalen Tieren hochgradig verändert. Vergleicht man die Dicke einzelner Lamellen, sind diese bei einem $10 \mathrm{~kg}$ schweren Mops doppelt so dick, wie die eines $40 \mathrm{~kg}$ schweren Schäferhundes [6].

- Thermoregulation: Die Hundenase hat zusätzlich zu den klassischen respiratorischen und olfaktorischen Aufgaben eine zentrale thermoregulatorische Funktion. Hunde können nicht schwitzen wie der Mensch, sie hecheln. Dabei wird, anders als oft irrtümlich angenommen, die Einatemluft über die Nase durch das feine Lamellensystem der großen ventralen Muschel geleitet [9] und erzeugt dort auf den über eine eigene Drüse (Gl. nasalis lateralis) befeuchteten Schleimhäuten sehr effektiv Verdunstungskälte. Diese Funktion ist bei brachyzephalen Tieren infolge der reduzierten nasalen Ventilation stark eingeschränkt und führt zu Wärmeintoleranz und verlängerten Erholungszeiten nach Belastung.

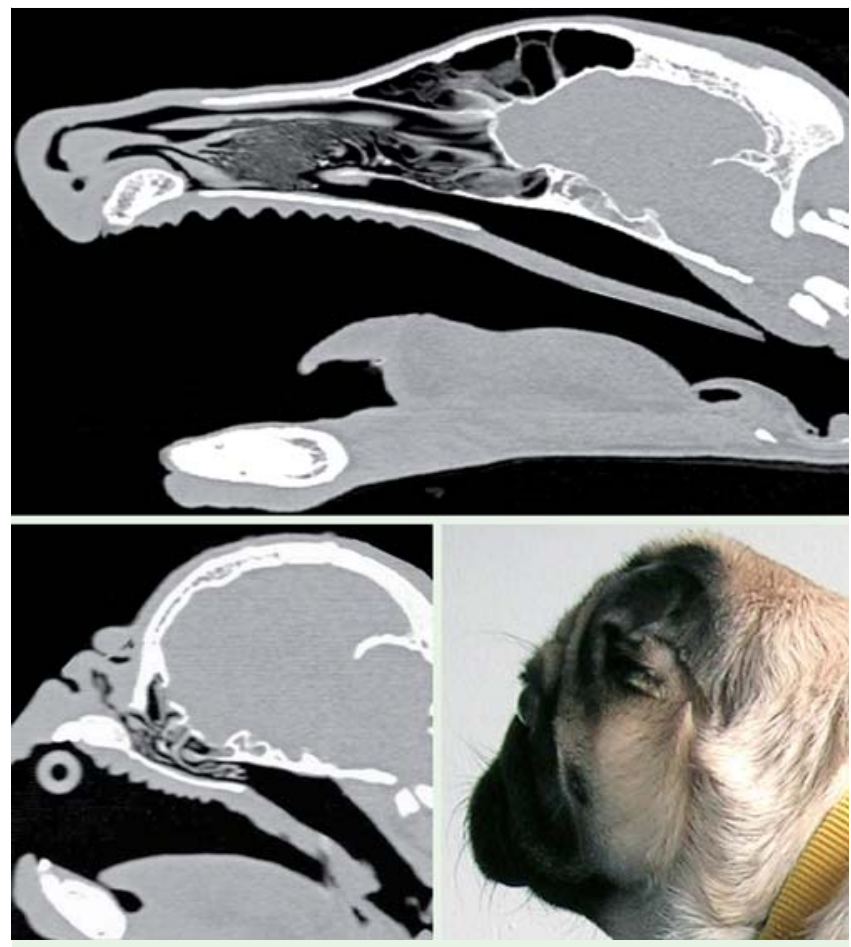

Abb. 1 Sagittales Computertomogramm des Hundeschädels [4]: oben normozephal (Schäferhund), unten brachyzephal (Mops). 


\section{Literatur}

1 Fournier PF. The Lorenz theory of beauty. J Cosmet Dermatol 2002; October 1 (3): 131-136

2 Zedda M, Manca P, Chisu Vet al. Ancient pompeian dogs - morphological and morphometric evidence for different canine populations. Anat Histol Embryol 2006; October 35 (5): 319-324

3 Riecks TW, Birchard SJ, Stephens JA. Surgical correction of brachycephalic syndrome in dogs: 62 cases (1991-2004). J Am Vet Med Assoc 2007; May 1, 230 (9): $1324-1328$

4 Oechtering TH, Oechtering GU, Nöller C. Strukturelle Besonderheiten der Nase brachyzephaler Hunderassen in der Computertomographie. Tieraerztl Prax 2007; 35 (K): 177-187

5 Hueber JP, Smith H-J, Reinhold P et al. Untersuchungen zur Geometrie und Funktion der Hundenase - Wie verändert sich die Funktion, wenn die Form verändert wird? Pneumologie 2010; 64 (Suppl.3): $\mathrm{S} 254$

6 Walter A, Seeger J, Oechtering GU, Nöller C. Dolichocephalic versus brachycephalic conchae nasales - a microscopic anatomical analysis in dogs. Anatomia Histologia Embryologia XXVII Congress of the Association of Veterinary Anatomists. Conference Proceeding. Budapest, Ungarn: 2008

7 Schlueter C, Budras KD, Ludewig E et al. Brachycephalic feline noses: CT and anatomical study of the relationship between head conformation and the nasolacrimal drainage system. J Feline Med Surg 2009; November 11(11): $891-900$

8 Oechtering GU, Hueber JP, Oechtering TH, Noeller C. Laser Assisted Turbinectomy (LATE) - Treating brachycephalic airway distress at its intranasal origin. Veterinary Surgery 2007; August 21, 36 (6): E18

9 Schmidt-Nielsen K, Bretz WL, Taylor CR. Panting in dogs: unidirectional air flow over evaporative surfaces. Science 1970; September 11, 169 (950): $1102-1104$

\section{Bibliografie}

DOI $10.1055 / \mathrm{s}-0030-1255513$

Pneumologie 2010; 64; 443-455

(c) Georg Thieme Verlag KG Stuttgart · New York

ISSN 0934-8387

\section{Korrespondenzadresse}

\section{Gerhard Oechtering}

Klinik für Kleintiere, Veterinärmedizinische Fakultät

der Universität Leipzig

An den Tierkliniken 23

04103 Leipzig

oechtering@kleintierklinik.uni-leipzig.de

\section{Geometrie und Funktion der Hunde-} nase: Wie ändert sich die Funktion, wenn die Form verändert wird?

\author{
J. P. Lippert ${ }^{1}$, P. Reinhold ${ }^{2}$, H. J. Smith ${ }^{3}$, P. Franco ${ }^{1}$, S. Y. Nather ${ }^{1}$, \\ C. Schlüter ${ }^{1}$, G. U. Oechtering ${ }^{1 *}$ \\ 1 Klinik für Kleintiere der Universität Leipzig \\ (Leiter: Prof. Dr. Gerhard Oechtering) \\ 2 Institut für molekulare Pathogenese im Friedrich-Loeffler-Institut \\ (Standort Jena) \\ 3 CareFusion; Jaeger (Höchberg)
}

\section{Zusammenfassung \\ $\nabla$}

Der intranasale Strömungswiderstand brachyzephaler Hunde liegt signifikant über dem normozephaler Hunde. Die nasale Resistance ist durch Xylometazolin kurzfristig und durch LATE-Therapie (Laser-assistierte Turbinektomie) langfristig um etwa 50\% absenkbar. Die Impuls-Oszillometrie stellt eine zuverlässige Methode zur quantitativen Erfassung intranasaler Stenosen der Hundenase dar und eignet sich zur Kontrolle des Therapieerfolgs. Mit Hilfe der akustischen Rhinometrie sind Veränderungen von Querschnitt und Volumen der Hundenase darstellbar. Ob eine räumliche Zuordnung von Stenosen in CT-Schnittbildern möglich ist, bleibt abzuwarten.

\section{Abstract \\ $\nabla$}

Nasal airflow resistance in brachycephalic dogs is significantly elevated compared to normal dogs. LaserAssisted TurbinEctomy (LATE)-surgery as well as xylometazolin were shown to reduce pathologically increased intranasal airway resistance in brachycephalic dogs by approximately $50 \%$. Impulse oscillometry provides a reliable and sensitive method to examine intranasal stenoses in the canine nose. Acoustic rhinometry allows assessment of changes in cross sectional area and volume of the canine nasal cavity.

Erkrankungen der Nase kommen bei Hunden relativ häufig vor und sind in der Regel mit einer funktionellen Beeinträchtigung verbunden. Neben der Atmung ist die Nase des Hundes für die Thermoregulation von zentraler Bedeutung. Vor dem Hintergrund, dass Hunde im Gegensatz zum Menschen obligate Nasenatmer sind [1,2], wird die große Bedeutung der nasalen Atmung ersichtlich.

In der Humanmedizin existiert eine Vielzahl an Methoden zur geometrischen Darstellung und funktionellen Quantifizierung nasaler Stenosen [3]. Die akustische Rhinometrie sowie die klassische Rhinomanometrie und Impuls-Oszillometrie werden beim Hund bisher nur im wissenschaftlichen Bereich angewendet. Dies zum einen als Tiermodell für Erkrankungen des Menschen $[4,5]$ und zum anderen bei der Erforschung von Erkrankungen der Tiere [6].

Ziel unserer Arbeit war es, die Impuls-Oszillometrie als funktionelle und die akustische Rhinometrie als Methode zur Darstellung der Nasengeometrie und Lokalisation der nasalen Stenosen bei Hunden mit anatomischen Veränderungen der Nase anzuwenden.

Bei der Anwendung sind anatomische Unterschiede zwischen der Nase des Menschen und der des Hundes zu bedenken. Beim Hund existiert eine Einengung im Nasenvorhof, die durch Verwendung eines röhrenförmigen Nasenadapters überbrückt werden kann. Die Verwendung eines solchen Nasenadapters ermöglicht es, den nasalen Strömungswiderstand unter Ausschluss der Nares (intranasaler Strömungswiderstand) zu erfassen. Somit ist der Einfluss von Strukturen innerhalb der Nasenhöhle - wie z. B. aberranten Conchen und Septumdeviationen - auf den Strömungswiderstand untersuchbar. Bei der Interpretation der Messergebnisse ist zu berücksichtigen, dass die Nasenmuscheln des Hundes komplex gebaut sind. Sie bilden ein Netzwerk aus Lamellen, die sich vielfach verzweigen. Bei brachyzephalen Hunden kommt es infolge von Malformationen der Nasenmuscheln zur intranasalen Obstruktion und Stenosierung des Nasenrachens [7]. Für den hier verwendeten Aufbau der Impuls-Oszillometrie ist es entscheidend, dass ein freier Druckausgleich zwischen Nasopharynx und Raumluft möglich ist. Aus diesem Grund

\footnotetext{
* Begründung für mehr als vier Autoren: Der Artikel gibt einen Einblick in verschiedene Untersuchungsmethoden, die Gegenstand von mehreren Studien sind.
} 

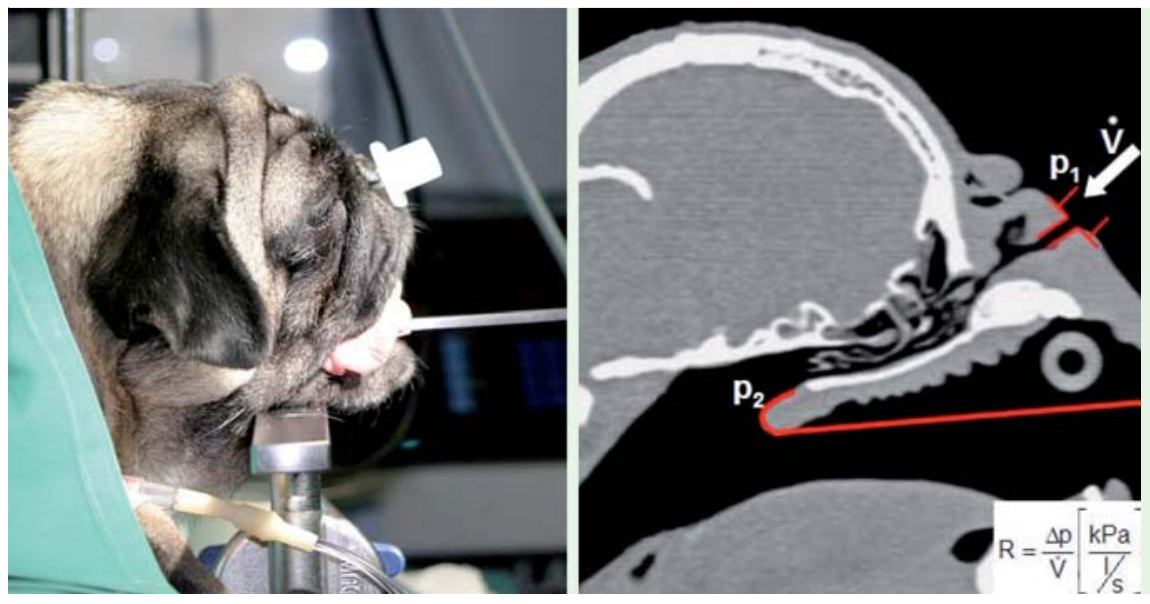

Abb. 1 Messaufbau für die impulsoszillometrische Messung des intranasalen Strömungswiderstandes am Hund (Foto [links] und Schema anhand eines CT-Bildes [rechts]): der in die Nasenöffnung eingeführte Tubuskonnektor dient als Adapter zwischen Messkopf und Nasenhöhle. Durch ein hakenförmiges Instrument wird das Gaumensegel nach rostral (anterior) gezogen und somit ein freier Druckausgleich zwischen Nasopharynx und Raumluft ermöglicht.

wurde das Gaumensegel mit einem hakenförmigen Instrument nach rostral (anterior) gezogen ( $\bullet$ Abb. 1$)$.

Vergleicht man den intranasalen Strömungswiderstand eines normozephalen Hundes (Beagle, $12 \mathrm{~kg}, \mathrm{R}=0,55 \mathrm{kPa} \mathrm{L}^{-1} \mathrm{~s}$ ) mit dem eines zur Laser-Assistierten Turbinektomie (LATE) [8] vorgestellten brachyzephalen Hundes (Mops, $10 \mathrm{~kg}, \mathrm{R}=1,3 \mathrm{kPa} \mathrm{L}^{-1} \mathrm{~s}$ ) ähnlicher Körpermasse, so wird deutlich, dass der intranasale Strömungswiderstand des kurznasigen Hundes deutlich über dem des nasengesunden Hundes liegt. Mit Hilfe der LATE-Therapie ließ sich dieser pathologisch erhöhte intranasale Strömungswiderstand um $50 \%$ auf $0,63 \mathrm{kPa} \mathrm{L}^{-1} \mathrm{~s}$ absenken. Die mit Hilfe der LATE-Therapie erreichte Vergrößerung des Nasenhöhlenquerschnitts ist ebenfalls mit der akustischen Rhinometrie darstellbar.

Betrachtet man den Einfluss des Schwellungszustandes der Nasenschleimhaut auf den intranasalen Strömungswiderstand, lässt sich Folgendes beobachten: durch Applikation des Dekongestivums Xylometazolin konnte der intranasale Strömungswiderstand beim normozephalen Hund (Beagle, 13,9 kg) von 0,56 auf $0,27 \mathrm{kPa} \mathrm{L}^{-1} \mathrm{~s}$ und beim brachyzephalen Hund (Mops, $7,0 \mathrm{~kg}$ ) von 1,13 auf $0,62 \mathrm{kPa} \mathrm{L}^{-1} \mathrm{~s}$ verringert werden. Der Widerstand war sowohl beim normozephalen als auch beim brachyzephalen Hund temporär etwa auf die Hälfte reduzierbar. Hierbei lag der Strömungswiderstand des brachyzephalen Hundes nach Verabreichung des Dekongestivums im physiologischen Bereich.

\section{Literatur}

1 Negus V. The Comparative Anatomy and Physiology of the Nose and Paranasal Sinuses. Edinburgh, London: E. \& S. Livingstone Ltd; 1958

2 Ohnishi T, Ogura JH, Nelson JR. Effects of nasal obstruction upon the mechanics of the lung in the dog. The Laryngoscope 1971; 2: 712-736

3 Grützenmacher S, Mlynski G, Mlynski B, Lang C. Die Objektivierung des Schwellungszustandes der Nasenschleimhaut - ein Vergleich von vier Messmethoden. Laryngo-Rhino-Otol 2003; 82: 645-649

4 Tiniakov RL, Tiniakova OP, McLeod RL et al. Canine model of nasal congestion and allergic rhinitis. J Appl Physiol 2003; 94 (5): 1821 - 1828

5 Nad N. Nasaler Widerstand beim Hund: Reproduzierbarkeit der Rhinomanometrie [Dissertation med. vet.] Zürich: Vetsuisse-Fakultät der Universität Zürich; 2004

6 Hueber JP. Impulsoszillometrische Untersuchung des intranasalen Atmungswiderstandes vor und nach laserassisstierter Turbinektomie zur Therapie des Brachyzephalen Atemnotsyndroms beim Hund [Dissertation med. vet.] Leipzig: Veterinärmedizinische Fakultät der Universität Leipzig; 2009 http://deposit.ddb.de/cgi-bin/dokserv? idn=994936559; Stand: Mai 2009

7 Oechtering TH, Oechtering GU, Nöller C. Strukturelle Besonderheiten der Nase brachyzephaler Hunderassen in der Computertomographie. Tieraerztliche Praxis 2007; 35 (K): 177-187
8 Oechtering GU, Hueber JP, Oechtering TH, Noeller C. Laser Assisted Turbinectomy (LATE) - Treating brachycephalic airway distress at its intranasal origin. Veterinary Surgery 2007; August 21, 36 (6): E18

\section{Bibliografie}

DOI $10.1055 / \mathrm{s}-0030-1255515$

Pneumologie 2010; 64; 443-455

(c) Georg Thieme Verlag KG Stuttgart · New York

ISSN 0934-8387

\section{Korrespondenzadresse}

\section{Dr. Johanna Lippert}

Klinik für Kleintiere der Universität Leipzig

An den Tierkliniken 23

04103 Leipzig

lippert@kleintierklinik.uni-leipzig.de

\section{Ausgewåhlte Verånderungen in den oberen Atemwegen des Pferdes - ein Überblick}

\section{K. Fey}

Klinik für Pferde, Innere Medizin, der Justus-Liebig-Universität Gießen

\section{Zusammenfassung \\ $\nabla$}

Pferde sind aufgrund ihrer obligaten Nasenatmung auf patente Nasengänge angewiesen.

Eine Vielzahl endoskopisch zu differenzierender dynamisch obstruktiver Veränderungen im pharyngealen und laryngealen Bereich kann verhindern, dass das Pferd als Athlet seine individuelle Höchstleistung erzielt. Die anatomische Besonderheit der Luftsäcke prädisponiert zu speziesspezifischen Erkrankungen.

\section{Abstract \\ $\nabla$}

Horses are obligate nasal breathers and depend on patency of their nasal passages.

Several dynamic obstructive diseases in the pharyngeal and laryngeal area can be differentiated by high speed treadmill endoscopy and may be responsible for impaired exercise tolerance in 
the equine athlete. The anatomical specialty of guttural pouches predisposes the horse to species-specific diseases.

Hinsichtlich der Funktion der oberen Atemwege des Pferdes sei zunächst betont, dass es sich bei Equiden um obligate Nasenatmer handelt. Ein langes Gaumensegel verhindert auch bei erheblicher Dyspnoe, dass diese Tiere durch die Mundhöhle atmen können. Daher ist die Durchgängigkeit der Nasengänge überlebensnotwendig.

Eine anatomische Besonderheit stellt das sogenannte falsche Nasenloch dar: als Verlängerung der ventralen Nasenmuschel bildet sich eine Flügelfalte aus, die medial zusammen mit der äußeren Haut eine ca. 8-10 cm lange, blind endende Nasentrompete des Pferdes formt. Als Schönheitsfehler, also ohne Verengung der Luftwege, kann es zu epithelialen Einschlusszysten kommen, die das kaudale Ende der Nasentrompete äußerlich vorwölben (๑ Abb. 1).

Die in der Ruhe umgekehrt kommaförmigen Nüstern werden bei forcierter Atmung durch Kontraktion der Nasenmuskulatur fast kreisrund geweitet. Ist die Flügelfalte verdickt, verbreitert oder wird sie nicht richtig angehoben, so wird sie bei körperlicher Belastung in die rostralen Atemwege eingesogen und verursacht einen Stridor. Ihre chirurgische Entfernung kann die Leistungsfähigkeit solcher Pferde verbessern [1].

Eine als speziesspezifisch beschriebene Neoplasie, das sogenannte progressive Siebbeinhämatom, geht typischerweise von der Submukosa der stark vaskularisierten ethmoidalen Turbinalien aus. Diese nicht metastasierende und nicht infiltrativ wachsende aber Nasengänge und teilweise Nebenhöhlen mechanisch verlegende - Umfangsvermehrung verursacht in ihrer Umgebung Drucknekrosen. Ein typisches klinisches Zeichen besteht in einem tröpfelnden blutigen Nasenausfluss. Es handelt sich nicht um einen soliden Tumor; nach chirurgischer Exzision entleert sich meist eine sero-sanguinöse Flüssigkeit. Da die Neoplasie aufgrund ihrer Lokalisation oft nur unvollständig entfernt werden kann, kommt es häufig zu Rezidiven. Eine Möglichkeit der chemischen Verödung besteht in der intratumoralen Injektion einer $4 \%$ igen gepufferten Formalinlösung [2].

Transnasal endoskopisch ist zuweilen eine sogenannte eingefangene Epiglottis auffällig. Dabei umfängt die physiologischerweise ventral des Kehldeckels liegende Schleimhaut diesen nach dorsal. Im endoskopischen Bild ist dann weder die knorpelige Umrandung noch die deutliche Gefäßzeichnung erkennbar, sondern ein wulstig erscheinender Schleimhautüberzug verdeckt zumindest die lateralen Anteile des Kehldeckels. Bei der Exspiration kann es zur Aufblähung dieser Schleimhautfalte und somit Verengung des Atemtraktes kommen. Häufiger verursacht die eingefangene Epiglottis allerdings eine Dysphagie mit Regurgitieren des Futters aus den Nüstern und die Futteraufnahme begleitende Hustenanfälle. Wichtigste Differenzialdiagnose stellt die Dorsalverlagerung des weichen Gaumens dar. Das lange Gaumensegel des Pferdes verdeckt dann die Epiglottis und es bildet sich eine V-förmige Falte. Diese Verlagerung kann intermittierend auftreten und wird häufig begleitend, z.B. bei den nicht seltenen entzündlichen Veränderungen in diesem Bereich, gesehen. Wie viele Einengungen im Bereich der oberen Atemwege wird die Dorsalverlagerung des Gaumensegels meist erst bei körperlicher Belastung symptomatisch. Vollblutpferde im Rennen erreichen Atemstromstärken von bis zu 75 l/s und inspiratorisch subatmosphärische Intrapleuraldrücke von $-30 \mathrm{~cm} \mathrm{H}_{2} \mathrm{O}$. Die nicht direkt Knorpel oder Knochen anliegenden pharyngealen und laryngealen Bereiche müssen aktiv weitgestellt bzw. unter Spannung gehal-

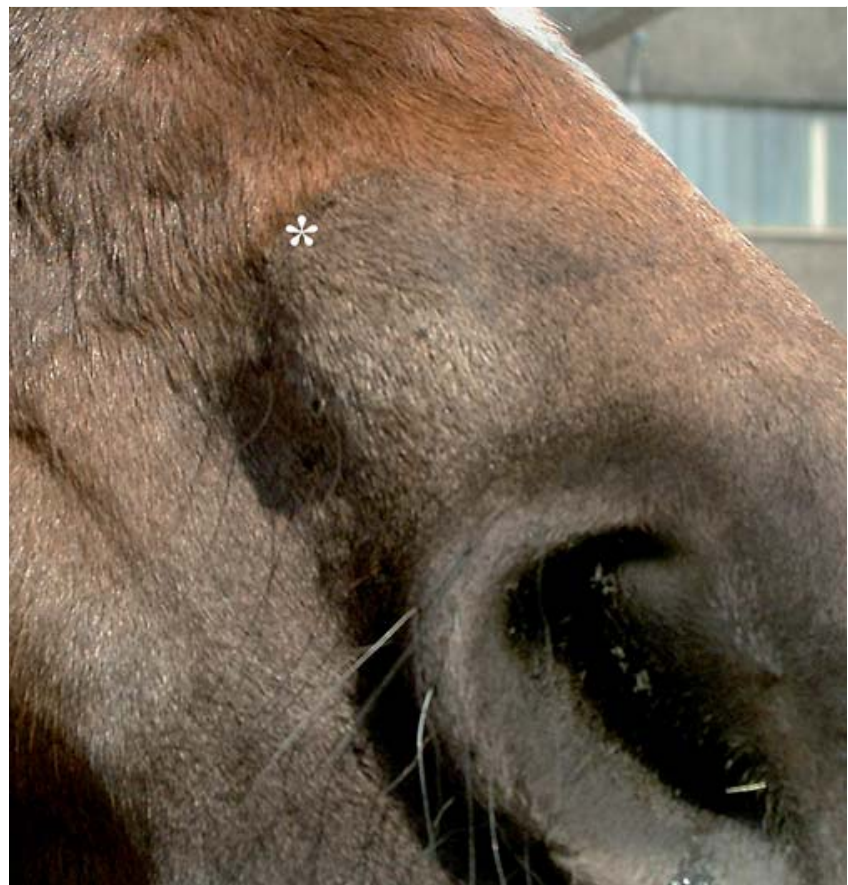

Abb. 1 Erkennbares kaudales Ende ( $\left.{ }^{*}\right)$ des falschen Nasenlochs eines Pferdes, da durch Atherom vorgewölbt.

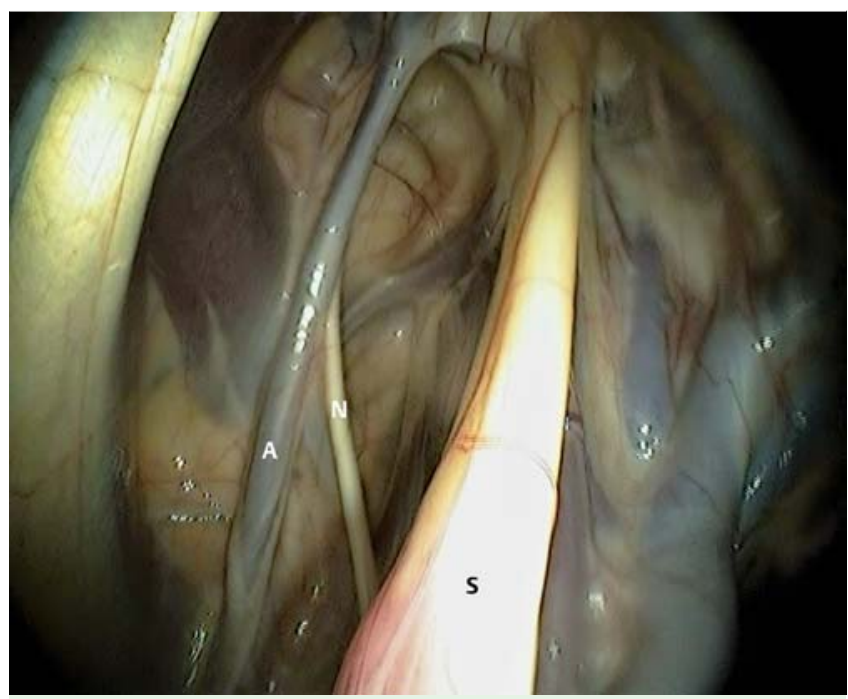

Abb. 2 Endoskopie eines linksseitigen Luftsacks eines Pferdes. S: Stylohyoid. A: Arteria carotis interna. N: Nervenfalte, in der u.a. N. vagus, N. glossopharyngeus und N. hypoglossus verlaufen.

ten werden, um eine optimale Luftpassage in die Trachea zu gewährleisten. Für die Diagnostik dynamischer Obstruktionen der oberen Atemwege werden Videos von Endoskopien genutzt, die während der körperlichen Belastung des Pferdes auf einem Laufband erstellt werden. Auch haben Endoskope, die während des Reitens am Kopf des Tieres fixiert sind und Bilder aus Rachenund Kehlkopfbereichen liefern, Marktreife erlangt. Eine Reihe von Kollapszuständen kann so differenziert werden. Im Fall der Dorsalverlagerung des weichen Gaumens kann es durch die belastungsbedingten tieferen subatmosphärischen Drücke bei der Inspiration zu einem Ansaugen des verlagerten Gaumensegels bis unter das Pharynxdach kommen [3]. Dies führt zu einem kompletten Verschluss des Atemwegs und damit zur Leistungsverweigerung des betroffenen Pferdes. 
Eine weitere häufige - und unter Umständen unter Belastung zu einem vollständigen Verschluss des Larynx führende - Veränderung ist nerval bedingt. Aus unbekannten Gründen verlieren dabei typischerweise die Axone des distalen Nervus laryngeus recurrens sinister ihre Myelinscheiden. Klinisch steht der Funktionsmangel des Musculus cricoarytenoideus dorsalis im Vordergrund: der wichtigste Abduktor des linksseitigen Aryknorpels atrophiert. Bereits in Ruhe kann dies zu einem in das Lumen der Rima glottis hängenden Aryknorpel führen. Oft sind solche Veränderungen aber nur unter forcierter Atmung zu erkennen dann kann der spannungslose linke Aryknorpel während der Inspiration soweit nach rechts verlagert werden, dass ein vollständiger Verschluss der Rima glottis resultiert. Aufgrund des bei Belastung entstehenden, oft charakteristisch pfeifenden, inspiratorischen Atemgeräusches wird diese Erkrankung auch als Kehlkopfpfeifen des Pferdes bezeichnet.

Als weitere anatomische Besonderheit der Equiden sei die starke Ausweitung der Ohrtrompete genannt, die als Luftsack (Diverticulum tubae auditivae) bezeichnet wird. Diese bilateral symmetrisch ausgeprägten Höhlen haben bei erwachsenen Pferden ein Volumen von jeweils ca. $500 \mathrm{ml}$. Ihr Sinn wird darin gesehen, die Kopfbeweglichkeit zu verbessern. Zudem wird diskutiert ob sie dazu beitragen, die bei körperlicher Hochleistung auf über $40^{\circ} \mathrm{C}$ erhöhte Bluttemperatur zu mindern. Da die Carotiden direkt unter der Schleimhaut verlaufen bevor sie in die Schädelhöhle eintreten, würde die Abkühlung des in ihnen pulsierenden Blutes durch die umgebende Luft einen Schutz des Gehirns vor Überwärmung bewirken. A. carotis interna, A. carotis externa und A. maxillaris verlaufen endoskopisch deutlich sichtbar direkt unter der Mukosa, ebenso wie N. vagus und für den Schluckakt und die Kehlkopffunktion wichtige Kopfnerven ( $\bullet$ Abb. 2).

Aufgrund ihrer Lokalisation sind diese Strukturen für Schädigungen infolge entzündlicher Erkrankungen der Schleimhaut prädisponiert. An erster Stelle steht hier die Luftsackmykose, bei der vorwiegend Aspergillen die Arterienwand infiltrieren und zu hochgradiger Epistaxis bis hin zum Verbluten führen können. Ligatur der betroffenen Arterien bzw. die Thrombosierung des geschädigten Arterienanteils über kathetergeleitete Platzierung von Embolisaten kann zur vollständigen Abheilung der Mykose führen [4]. Befallen die Pilze die subepithelial verlaufenden Nerven, so ist das klinische Zeichen meist eine Dysphagie.

Da die in den Nasopharynx mündenden Luftsackklappen oberhalb des tiefsten Punktes der Luftsacklumina liegen, sammelt sich entzündliches Sekret hier oftmals an. Ein Luftsackempyem bildet sich aufgrund fortgeleiteter bakterieller Infekte der respiratorischen Mukosa des Nasopharynx oder nach Abszedierung des retropharyngealen Lymphknotens in den Luftsack hinein. Für diese Lymphadenitis ist meist Streptococcus equi subspezies equi verantwortlich, der Erreger der Druse des Pferdes. Bei Fütterung vom Boden wird die Entleerung des Luftsackes erleichtert, da bei tiefer Kopfposition die Luftsacköffnung relativ zum Luftsackboden günstiger liegt und sich beim Schluckakt die Luftsackklappen öffnen. Verbleibt ein Empyem über längere Zeit, so können Konkremente entstehen, die endoskopisch mittels Körbchen oder offen chirurgisch entfernt werden müssen.

Bei der Luftsacktympanie kommt es infolge einer genetischen Disposition [5] zu einer fehlerhaften Ausprägung der Luftsackklappe. Bei dieser Erkrankung kann die Luft den Luftsack aber nicht mehr verlassen, und er bläht sich nach außen sichtbar immer mehr auf. Meist zeigt sich dann bereits im Fohlenalter äußerlich eine prall luftgefüllte Umfangsvermehrung zwischen Unterkiefer und Hals, die durch innere Kompression des Pharynx und der oberen Trachea zu hochgradiger Dyspnoe führen kann. Da die Klappen meist unterschiedlich stark verändert sind, ist das klinische Bild oft einseitig ausgeprägt. Eine elegante Therapie besteht in der Laser-endoskopischen Fensterung der medianen Lamelle zwischen den beiden Luftsäcken, so dass die Luft über die nicht oder weniger betroffene Seite entweichen kann [6].

\section{Literatur}

1 Hawkins JF, Tulleners EP, Evans LH, Orsini JA. Alar fold resection in horses: 24 cases (1979-1992). J Am Vet Med Assoc 1995; 206: 1913 1916

2 Conti MB, Marchesi MC, Rueca F, Puccetti M. Diagnosis and treatment of Progressive Ethmoidal Haematoma (PEH) in horses. Vet Res Commun 2003; 27 Suppl. 1: 739 - 743

3 Holcombe SJ, Ducharme NG. Disorders of the nasopharynx and soft palate.In: McGorum BC, Dixon PM, Robinson NE, Schumacher J, eds. Equine Respiratory Medicine and Surgery. Philadelphia: Saunders Elsevier; 2007: 437-457

4 Schneider M, Fey K, Tellhelm B et al. Perkutaner Verschluss arterieller Gefäße durch permanente Embolisate zur Therapie einer Luftsackblutung beim Pferd. Tierärztliche Praxis 1998; $26(G): 211-215$

5 Zeitz A, Spötter A, Blazyczek I et al. Whole-genome scan for guttural pouch tympany in Arabian and German warmblood horses. Anim Genet(epub) PMID 19703122. 24 Aug 2009

6 Blacyczek I, Hamann H, Deegen E et al. Retrospective analysis of 50 cases of guttural pouch tympany in foals. Vet Rec 2004; 154: 261 - 264

\section{Bibliografie}

DOI $10.1055 / s-0030-1255516$

Pneumologie 2010; 64; 443-455

(c) Georg Thieme Verlag KG Stuttgart · New York

ISSN 0934-8387

\section{Korrespondenzadresse}

PD Dr. Kerstin Fey

Klinik für Pferde, Innere Medizin

Frankfurter Str. 126

35392 Gießen

Kerstin.Fey@vetmed.uni-giessen.de 